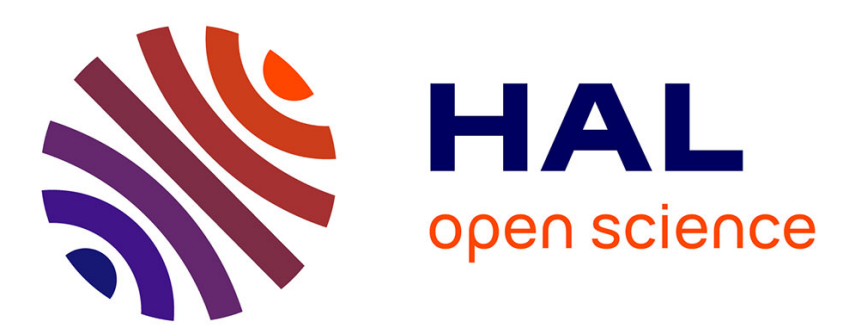

\title{
Coexistence de deux types de modulations dans des échantillons trempés du supraconducteur $\mathrm{BiSrCaCuO}$
} (2212)

\author{
R. Mokrani, M. Ben Salem, O. Monnereau, F. Remy, G. Vacquier, C.
} Boulesteix

\section{To cite this version:}

R. Mokrani, M. Ben Salem, O. Monnereau, F. Remy, G. Vacquier, et al.. Coexistence de deux types de modulations dans des échantillons trempés du supraconducteur BiSrCaCuO (2212). Revue de Physique Appliquée, 1990, 25 (1), pp.45-48. 10.1051/rphysap:0199000250104500 • jpa-00246160

\section{HAL Id: jpa-00246160 https://hal.science/jpa-00246160}

Submitted on 1 Jan 1990

HAL is a multi-disciplinary open access archive for the deposit and dissemination of scientific research documents, whether they are published or not. The documents may come from teaching and research institutions in France or abroad, or from public or private research centers.
L'archive ouverte pluridisciplinaire HAL, est destinée au dépôt et à la diffusion de documents scientifiques de niveau recherche, publiés ou non, émanant des établissements d'enseignement et de recherche français ou étrangers, des laboratoires publics ou privés. 
Classification

Physics Abstracts

$61.14-74.10-74.70$

\title{
Coexistence de deux types de modulations dans des échantillons trempés du supraconducteur BiSrCaCuO (2212)
}

\author{
R. Mokrani ( $\left.{ }^{1}\right)$, M. Ben Salem ( $\left.{ }^{1}\right)$, O. Monnereau ( $\left.{ }^{2}\right)$, F. Remy $\left({ }^{2}\right)$, G. Vacquier $\left({ }^{2}\right)$ \\ et C. Boulesteix ( $\left.{ }^{1}\right)$ \\ (1) Lab. de Microscopie Electronique U.A. 797, Fac. Sci. St-Jérôme, av. Escadrille Normandie-Niemen, \\ 13397 Marseille Cedex 13, France \\ (2) Lab. de Chimie des Matériaux, Université de Provence, 3 Place Victor Hugo, 13331 Marseille Cedex 03, \\ France
}

(Reçu le 28 mai 1989, révisé le 25 septembre 1989, accepté le 9 octobre 1989)

\begin{abstract}
Résumé. - Kang et al. ont montré [1] que la modulation de $\mathrm{BiSrCaCuO}$ (2212) le long de l'axe $b^{*}$ peut être de 3 sortes : M1, M2, M3. Cette modulation peut être reliée aux conditions de préparation et à la valeur de $T_{\mathrm{c}}$ du supraconducteur. On montre ici que M1 et M3 peuvent coexister dans un même cristal dans le cas d'échantillons trempés, ce qui correspond à l'empilement de différentes couches modulées suivant les types M1 et M3. Ces deux modulations différentes peuvent se produire soit suivant la même direction, soit suivant deux directions perpendiculaires.
\end{abstract}

\begin{abstract}
It has been shown by Kang et al. [1] that the modulation of $\mathrm{BiSrCaCuO}$ (2212) compounds along the $b^{*}$ axis can be of 3 different kinds : M1, M2, M3. This modulation can be related to the preparation conditions and to the $T_{\mathrm{c}}$ value of the superconductor. It is shown here that M1 and M3 can occur in the same crystal in the case of quenched samples, which corresponds to the stacking of different layers modulated as M1 and M3. The two different modulations may occur either along the same direction, or in two perpendicular directions.
\end{abstract}

\section{Introduction.}

Kang et al. ont montré dans un article récent [1] que la modulation de composés $\mathrm{BiSrCaCuO}$ de composition nominale (2212) le long de l'axe $b^{*}$ peut être de 3 sortes, appelées M1, M2, M3 par les auteurs. Ces modulations sont incommensurables. La première modulation M1 correspond sensiblement à $q^{*}=$ $2 b^{*} / 5$, tandis que les deux autres M2 et M3

$b^{*} / 5$. La différence entre M2 et M3 réside dans l'intensité des satellites : pour M2, seul le premier satellite est intense, tandis que pour M3 plusieurs satellites sont intenses. Ainsi la différence entre M1 et les deux autres est quantitative alors qu'elle est seulement qualitative entre M2 et M3. Les conditions d'apparition de ces différentes modulations dépendent des conditions de préparation des échantillons, comme cela a été montré précédemment $[1,2]$. On peut les rencontrer toutes les trois dans des petits cristaux obtenus à partir du composé partiellement fondu et refroidi lentement [1] ou dans des céramiques recuites entre 820 et $880^{\circ} \mathrm{C}$ [1]. Dans les échantillons trempés qui ont été étudiés ici, M2 est pratiquement absent, on ne s'intéressera donc qu'à M1 et M3. La figure 1a, b représente les deux types de modulation M1 et M3. Il est à noter que ces deux modulations sont aussi celles décrites par Matsui et al. [3], mais pour une composition déterminée par microanal se ui serait un eu différente (4336).

On sait que les oxydes mixtes $\mathrm{BiSrCaCuO}$ synthétisés et étudiés par [4-6] présentent trois phases supraconductrices, avec des $T_{\mathrm{c}}$ de $20 \mathrm{~K}, 90 \mathrm{~K}, 110 \mathrm{~K}$ environ. La seconde phase $(90 \mathrm{~K})$ correspond à la composition (2212), qui est la composition moyenne attendue de nos échantillons. [1] a montré par ailleurs que pour cette composition des petites variations de $T_{\mathrm{c}}$ (de 86 à $94 \mathrm{~K}$ ) existent. Elles sont liées au type de modulation rencontrée, la modulation M1 correspondant alors à la valeur de $T_{\mathrm{c}}$ la plus élevée, ceci est en accord avec d'autres résultats [7]. 


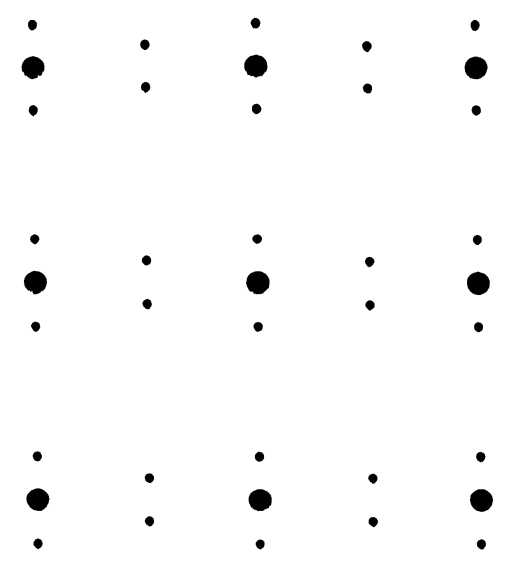

a)

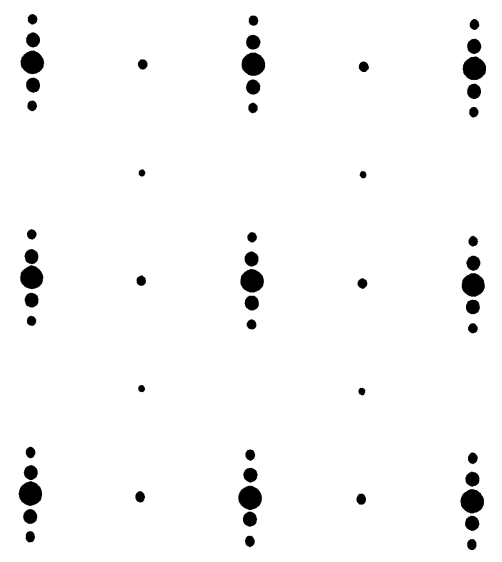

b)

Fig. 1. - Schéma des modulations M1 (a) et M3 (b).

[Schematic representation of modulations M1 (a) and M3 (b).]

D'autre part il est reconnu que le comportement du matériau obtenu est dépendant du processus expérimental (traitement thermique et nature des réactifs utilisés). Ainsi une variation de $T_{\mathrm{c}}$ similaire à celle qui est signalée par [1] a été observée par Zhao et al. [8] : elle serait dépendante du cycle thermique et de l'atmosphère du recuit et correspondrait d'après les auteurs à une faible variation de masse qui serait liée à une variation de la teneur en oxygène. Enfin pour la composition (2212), de nombreux auteurs signalent de faibles raies de diffraction $\mathrm{X}$ non indexées, traduisant le caractère polyphasé du produit. Sastry et al. [9] et Beltran et al. [10] ont pu toutefois obtenir une seule phase en faisant intervenir un précurseur du type (0212) pour le premier et (2001) pour le second.

Dans l'étude décrite ici, les échantillons de composition nominale (2212) ont d'abord été recuits à température bien définie puis trempés à l'ambiante. On a noté chez ces échantillons des comportements nouveaux en ce qui concerne la modulation suivant $b^{*}$.

\section{Processus de préparation des échantillons.}

Les composés $\mathrm{Bi}_{2} \mathrm{O}_{3}(3 \mathrm{~N}), \mathrm{SrCO}_{3}(4 \mathrm{~N} 5), \mathrm{CaCO}_{3}$ (4N5) et $\mathrm{CuO}(5 \mathrm{~N})$ sont intimement mélangés en proportion stœechiométrique $2,2,1,2$ et portés à $800^{\circ} \mathrm{C}$ pendant deux jours. Ce premier traitement thermique est suivi d'un broyage, puis d'un deuxième traitement à $840^{\circ} \mathrm{C}$ durant une semaine entrecoupé de broyages supplémentaires et d'un pastillage. Tous les traitements sont effectués à l'air sous pression atmosphérique. Le spectre $X$ obtenu à l'issue du dernier traitement présente essentiellement les raies de la phase (2212). Une série de raies faibles non indexées est également présente. Ces raies ne sont pas attribuables aux raies induites par la modulation : elles correspondent à une seconde phase très fortement minoritaire.
L'oxyde ainsi obtenu subit alors un recuit de trois à dix jours en nacelle d'or à une température inférieure à $890^{\circ} \mathrm{C}$, suivi d'une trempe à l'air. En opérant ainsi, on conserve la phase (2212) comme le montre la diffraction $\mathrm{X}$, qui par contre commence à disparaître au-dessus de $890^{\circ} \mathrm{C}$ [11]. On peut ainsi considérer que les échantillons étudiés ici sont très majoritairement constitués de la phase (2212). Bien que l'existence d'autres phases ne soit pas totalement exclue, on peut supposer que les différentes modulations que l'on observe par diffraction électronique correspondent toutes à la phase (2212) avec des écarts possibles à la stœchiométrie comme le confirme la microanalyse.

\section{Microanalyse et modulation des échantillons trempés.}

La microanalyse et les études de microscopie électronique ont été effectuées sur des échantillons recuits à $880^{\circ} \mathrm{C}$ et trempés. La microanalyse confirme la très large prédominance de la phase (2212) mais avec des écarts de stœchiométrie parfois importants. On a obtenu comme composition caractéristique: $\mathrm{Bi}$ $28 \%, \mathrm{Sr} 31 \%, \mathrm{Ca} 12 \%, \mathrm{Cu} 29 \%$, ce qui correspond assez bien à (2212). Mais exceptionnellement on a pu rencontrer des compositions du type $(2 ; 3,5 ; 1$; $0,3)$ et $(4 ; 2 ; 0,5 ; 2)$. Par ailleurs, des traces d'oxyde de calcium n'ayant pas réagi ont été rencontrées parfois ce qui explique une déficience possible en calcium.

La modulation observée est de type M3. Toutefois pour la première fois une double modulation M1 et M3 a été observée (Fig. 2). Le voisinage ou la superposition de deux domaines d'un cristal dont les modulations sont orientées à $90^{\circ}$ est fréquente pour les cas M2 ou M3 [2, 12]. Une double modulation aurait été observée par $\mathrm{Li}$ et al. [13] pour le cas de M1 mais il pourrait s'agir là encore de deux domaines à $90^{\circ}$. La figure 2 montre qu'un même cristal peut présenter les deux modulations M1 et M3 à $90^{\circ}$. Les 


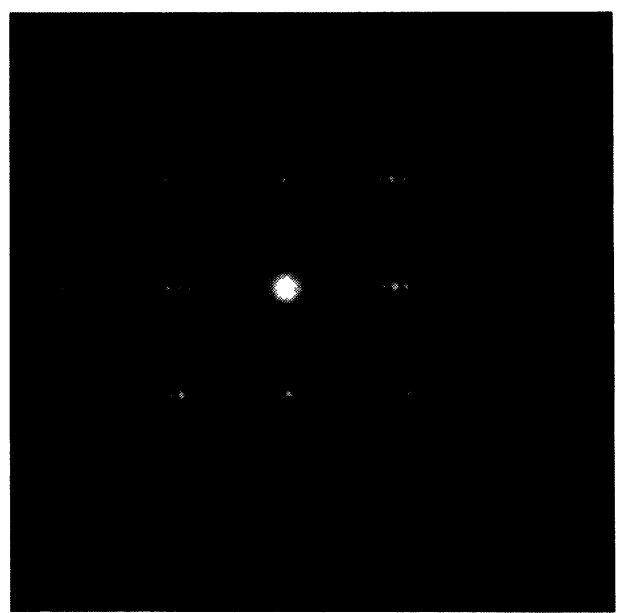

Fig. 2. - Diffraction d'un cristal présentant une superposition des deux modulations M1 et M3 à $90^{\circ}$.

[Diffraction given by a crystal with an overlay of M1 and M3 rotated by $90^{\circ}$.]

taches de modulation de M1 étant dans ce cas ellesmêmes modulées par M3, il est clair que la modulation se fait ici par feuillets successifs M1, M3, et non pas par voisinage de deux régions de modulations différentes. La figure 3 où le second satellite est plus intense que le premier (situation observée par Hewat [14]) correspondrait alors à la superposition des deux modulations dans la même direction. Les modulations M1 et M3 ont été observées par de nombreux auteurs et dans la très grande majorité des cas pour des compositions (2212); de plus, la très grande majorité de nos échantillons correspond à cette composition. Nous pouvons donc raisonnablement admettre que les deux types de modulations correspondent l'une et l'autre à la composition (2212). Les travaux de [15] reliant la valeur de $T_{\mathrm{c}}$ au traitement thermique et à la teneur en oxygène et ceux de [1] reliant la modulation au traitement thermique et à la valeur de $T_{\mathrm{c}}$ laisseraient supposer

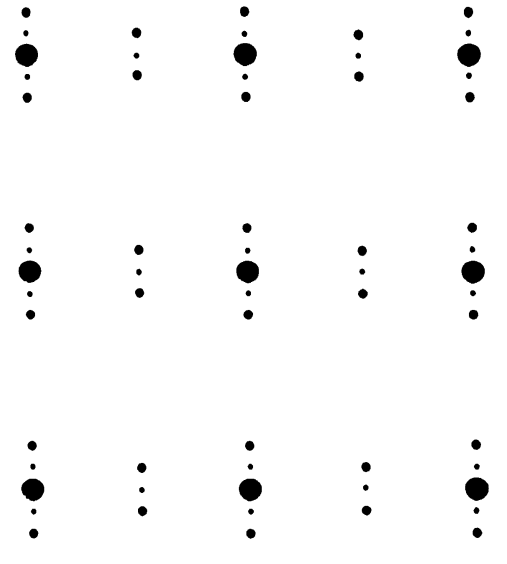

Fig. 3. - Schéma de la superposition de deux modulations M1 et M3 suivant la même direction.

[Schematic representation of an overlay of modulations M1 and M3 occurring along the same direction.]

que le degré d'oxydation pourrait être directement lié à la modulation observée, mais d'autres hypothèses existent reliant les différentes modulations observées à des variations de composition (cations) [1, 16]. De telles variations locales de composition ont été observées sur nos échantillons trempés et nous n'avons pas actuellement d'élément probant permettant de choisir entre l'une ou l'autre hypothèse.

\section{Conclusion.}

On a montré qu'il y a possibilité de coexistence dans un cristal de $\mathrm{BiSrCaCuO}$ (2212) de deux modulations de types M1 et M3 suivant deux directions perpendiculaires. L'existence de différents types de modulations dans des directions parallèles expliquerait alors la forme de certains types de clichés de diffraction rencontrés dans la littérature.

\section{Bibliographie}

[1] Kang Z. C., Monnereau O., Remy F., Spas S., Casalot A., Sorbier J. P., Fournel A. and BoulesteIX C., J. Phys. France 50 (1989) 1227.

[2] Kang Z. C., Morin D., Monnereau O., BoulesTEIX C., Phys. Status Solidi a 113 (1989) 23.

[3] Matsui Y., Maeda H., TANaKa Y. and HoRiuchi S., Jpn J. Appl. Phys. 27 (1988) L361.

[4] Michel C., Hervieu M., Borel M., Grandin A., Deslandes F., Provost J. et Raveau B., $Z$. Phys. B 68 (1987) 421.

[5] Maeda H., Tanaka Y., Fukutomi M., Asano T., Jpn J. Appl. Phys. 27 (1988) L209.
[6] Tarascon J. M., Le Page Y., Barboux P., BAgley B. G., GreEnE L. H., MCKINNON W. R., Hull G. W., Giroud M., Hwang D. M., Phys. Rev. B 37 (1988) 9382.

[7] Shishido T., Shindo D., UKel K., SASAKi T., Toyota N. and Fukuda T., Jpn J. Appl. Phys. 28 (1989) L791.

[8] Zhao J., Seehra M. S., Physica $C$, à paraître.

[9] Sastry P., Gopalakrishnan I. K., Sequeira A., Rajagopal H., Gangadharan K., Phatak G. M., IYER R. M., Physica C 156 (1988) 230. 
[10] Beltran D., Caldes M. T., Ibanez R., Martinez E., Escriva E., Beltran A., J. Less. Common Met. 150 (1989) 247.

[11] MONNEREAU O. et al. (à paraître).

[12] Van Tendeloo G., Zandbergen H. W., VAN Landuyt J., Amelinckx S., Appl. Phys. A 45 (1988) 748 ; Appl. Phys. A 46 (1988) 153.

Van Tendeloo G., Zandbergen H. W., AmeLINCKX S., Solid State Commun. 66 (1988) 927.
[13] Li G. Q., Chen C., YANG D., Li F. H., YAO Y. S., RAN Z. Y., WANG W. K. et ZHAO Z.X., Z. Phys. B Condensed Matter 74 (1989) 165.

[14] Hewat E., Colloque A.R.C. microstructure des supraconducteurs à haute température critique, Giens (1989).

[15] Zhao J. and Seehra M., Physica $C$ (à paraître).

[16] Casanove J., Baules P., SnOeck E. and Roucau C., Physica C 159 (1989) 461. 